\title{
Foxk1 regulates cancer progression
}

\author{
Daniel J. Garry ${ }^{1,2,3,4}$, Geunho Maeng ${ }^{1}$, Mary G. Garry ${ }^{1,2}$ \\ ${ }^{1}$ Lillehei Heart Institute, Department of Medicine, ${ }^{2}$ Paul and Sheila Wellstone Muscular Dystrophy Center, ${ }^{3}$ Stem Cell Institute, ${ }^{4}$ Masonic Cancer \\ Center, University of Minnesota, Minneapolis, MN, USA \\ Correspondence to: Daniel J. Garry, MD, PhD. Lillehei Heart Institute, 2231 6th St SE, (CCRB 4-146), University of Minnesota, Minneapolis, MN \\ 55455, USA. Email: garry@umn.edu. \\ Provenance and Peer Review: This article was commissioned by the editorial office, Annals of Translational Medicine. The article did not undergo \\ external peer review. \\ Comment on: Wang Y, Qiu W, Liu N, et al. Forkhead box K1 regulates the malignant behavior of gastric cancer by inhibiting autophagy. Ann Transl \\ Med 2020;8:107.
}

Submitted Apr 15, 2020. Accepted for publication Jun 05, 2020.

doi: 10.21037/atm-2020-94

View this article at: http://dx.doi.org/10.21037/atm-2020-94

Cancer remains a major cause of morbidity and mortality. The lifetime risk for developing cancer is one in three and approximately 1.7 million new cases of cancer were diagnosed in the U.S. in 2019 according to the American Cancer Society (1). Worldwide, gastric carcinoma (GC) is the fourth most common malignancy and is the second leading cause of death $(1,2)$. GC is a malignant disease and is associated with a poor long-term prognosis $(1,2)$. Therefore, new therapies are warranted, which require an enhanced understanding of the mechanisms that govern gastric cancer progression. Previous in vitro studies have defined pathways (sonic hedgehog, stem cell signaling, cell cycle, DNA damage, Notch, PI3K/AKT, Tgfb, Wnt, etc.) and transcription factors (TP53, EGR1, GATA, GLI, STAT, MYC/MAX, SMAD2/SMAD3/SMAD4) that are expressed in gastric cancer cell lines (3). One pathway that has received intense interest is the forkhead/windged helix transcription factor family.

Forkbead was initially discovered in the fly and disruption of the gene resulted in a forked head phenotype (4). More than 100 members have been assigned to the family based on the homology of the 100 amino acid containing DNA binding domain (the forkhead/windged helix or Fox domain) (5-7). These family members function as transcription factors to regulate cell and lineage specification during development, metabolism, aging or survival, stem cell populations, tissue repair, diseases and others (5-7). For example, Foxd3 (Genesis) has been shown to be expressed in embryonic stem cells (8) whereas Foxb1 has been shown to be a regulator of neural progenitors (9) and Foxm1 has been shown to be expressed in regenerating hepatocytes (10). In addition, Foxo factors have been shown to inhibit apoptosis, regulate PI3K signaling and serve as an anti-aging factor (7). More recently, members of this family have been shown to function as pioneer factors as they have the ability to bind nucleosomal DNA and unwrap the chromatin thereby exposing DNA binding motifs for the binding of other transcription factors and subsequent regulation of gene expression $(8,9)$. In addition to this array of molecular and cellular functional roles, this family also regulates cell cycle kinetics. Foxk1 has been shown to be an essential regulator of cell proliferation.

Foxk1 was initially termed MNF (Myocyte Nuclear Factor) based on the restricted expression pattern in the myogenic lineages during murine embryogenesis and was discovered in the Williams' laboratory $(10,11)$. Structurally, Foxk1 harbors motifs that have important functional roles including: the leucine zipper motif, the $\operatorname{Sin} 3$ interacting motif (SIM) domain, and the Forkhead-associated (FHA) domain, which increase the complexity regarding its functional role(s), the winged helix DNA binding domain (WHD) and the transcriptional activation domain (TAD) (Figure 1A) (12,13). For example, Foxk1 is the prototype for the FHA domain, which is a phosphopeptide-binding motif that functions to recruit interacting proteins and regulate cell cycle kinetics (14). Previous studies have demonstrated that Foxk1 and its interacting partners (Fhl2, Sds3 and others) function to repress the $p 21$ gene thereby promoting 

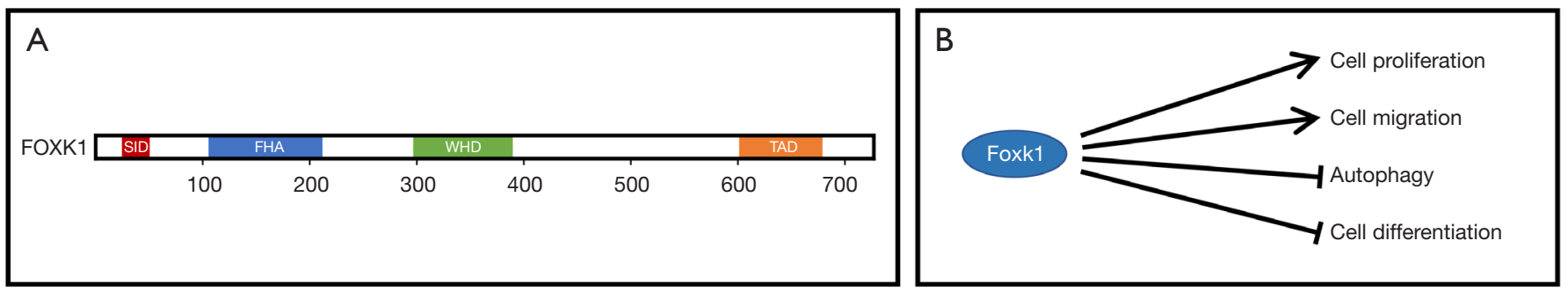

Figure 1 Foxk1 is an important regulator in stem cell and cancer cell populations. (A) Schematic highlighting the domains of the FOXK1 protein. The Sin3 interacting domain (SID) physically interacts with Sin3 and its associated complex. The Forkhead-Associated Domain (FHD) interacts with phosphothreonine proteins (such as SDS3) and is important in the regulation of cell cycle kinetics. The winged helix domain (WHD) binds to DNA and allows FOXK1 to function as a transcriptional regulator. The transcriptional activation domain (TAD) is located in the carboxy terminal region of the protein. (B) FOXK1 has been shown to have a number of permissive roles and repressive roles in stem cell and cancer cell populations.

cell proliferation and repressing lineage differentiation (Figure 1B) (14-17). Using gene disruption technology, Foxk1 null mice have been shown to be partially lethal and the limited null mice that survived were growth retarded and had impairments in tissue regeneration (18). Studies have also shown an increase of Foxk1 expression associated with a number of malignancies including: melanoma, breast, pancreatic, osteosarcoma, glioblastoma, ovarian, esophageal, prostate and gastric cancers (19-24).

The role of Foxk1 and GC was further examined in the recent study by Wang et al. (25). These investigators used computational biology, western blot analysis and tissue microarrays to define FoxK1 expression in GC cell lines and human gastric cancer specimens. Not only did they verify an induction of Foxk1 expression associated with GC (compared to controls) but they also observed a correlation between those having increased Foxk1 expression and malignant progression of GC (25). Next, using in vitro assays, overexpression or siRNA knockdown strategies and EdU incorporation Wang et al., demonstrated that Foxk1 increased GC cell proliferation, migration and invasion (Figure 1B) (25). Importantly, they further demonstrated that increased Foxk1 expression portended a poor prognosis for patients with GC. Wang et al., then established the mechanistic role for increased Foxk1 expression and GC as Foxk1 was shown to suppress autophagy in GC via the $\mathrm{PI} 3 \mathrm{~K} / \mathrm{AKT} / \mathrm{mTOR}$ pathway (Figure 1B) (25).

The study by Wang et al., has a number of important findings. First, they used human in vitro (GC cell lines) and in vivo (GC samples) specimens. Second, they used a large number of GC samples (43 pairs of GC and control or nonGC specimens). Third, their results supported the notion that Foxk1 functioned in the context of GC as a repressor of autophagy via the PI3K/AKT/mTOR pathway (25). This study provides the rationale for using Foxk1 as a molecular marker for progression of GC and response to treatment. Moreover, based on this study, Foxk1 may be an important target for GC treatment. Therefore, small molecule or chemical genetics strategies may be employed, in the future, to identify Foxk1 specific inhibitors that may be used in combination with surgical debulking or chemotherapies in patients with GC. In some respects, the findings by Wang et al., are somewhat predictable. This is, in part, due to the previous studies that have conclusively shown that Foxk1 directly interacts with Fhl2 (12), promotes cell proliferation (12-18), has increased expression in other human cancers including GC and functions to regulate autophagy (19-24). Nevertheless, if these studies further establish a role for Foxk1 as a regulator of GC malignant progression and ultimately lead to effective therapeutics then the medical impact will be significant.

In summary, Foxk1 is an important regulator of cell proliferation, quiescence and differentiation in stem cell populations and cancer. The studies by Wang et al., provide an important platform to decipher putative inhibitors of Foxk1 with the goal of bending the malignancy outcome curve for GC.

\section{Acknowledgments}

The authors acknowledge the laboratory of R. Sanders Williams and their initial studies and contributions focused on MNF (Foxk1). The authors further acknowledge all the graduate students, technicians and postdoctoral fellows that 
have contributed to our knowledge of Foxk1 biology in the Garry laboratory. We also want to recognize all those investigators and their laboratorios that have contributed to the field of study focused on Fox transcription factors and were not referenced in this manuscript due to page limitations.

Funding: None.

\section{Footnote}

Conflicts of Interest: All authors have completed the ICMJE uniform disclosure form (available at http://dx.doi. org/10.21037/atm-2020-94). DJG and MGG report other from NorthStar Genomics, outside the submitted work. GM has no conflicts of interest to declare.

Ethical Statement: The authors are accountable for all aspects of the work in ensuring that questions related to the accuracy or integrity of any part of the work are appropriately investigated and resolved.

Open Access Statement: This is an Open Access article distributed in accordance with the Creative Commons Attribution-NonCommercial-NoDerivs 4.0 International License (CC BY-NC-ND 4.0), which permits the noncommercial replication and distribution of the article with the strict proviso that no changes or edits are made and the original work is properly cited (including links to both the formal publication through the relevant DOI and the license). See: https://creativecommons.org/licenses/by-nc-nd/4.0/.

\section{References}

1. Siegel RL, Miller KD, Jemal A. Cancer statistics, 2019. CA Cancer J Clin 2019;69:7-34.

2. Sitarz R, Skierucha M, Mielko J, et al. Gastric cancer: epidemiology, prevention, classification, and treatment. Cancer Manag Res 2018;10:239-48.

3. Periasamy J, Muthuswami M, Rao DB, et al. Stratification and delineation of gastric cancer signaling by in vitro transcription factor activity profiling and integrative genomics. Cell Signal 2014;26:880-94.

4. Jurgens G, Weigel D. Terminal versus segmental development in the Drosophila embryo: the role of the homeotic gene fork head. Roux Arch Dev Biol 1988;197:345-54.

5. Golson ML, Kaestner KH. Fox transcription factors: from development to disease. Development 2016;143:4558-70.
6. Hannenhalli S, Kaestner KH. The evolution of Fox genes and their role in development and disease. Nat Rev Genet 2009;10:233-40.

7. Myatt SS, Lam EW. The emerging roles of forkhead box (Fox) proteins in cancer. Nat Rev Cancer 2007;7:847-59.

8. Iwafuchi-Doi M, Donahue G, Kakumanu A, et al. The Pioneer Transcription Factor FoxA Maintains an Accessible Nucleosome Configuration at Enhancers for Tissue-Specific Gene Activation. Mol Cell 2016;62:79-91.

9. Iwafuchi M, Cuesta I, Donahue G, et al. Gene network transitions in embryos depend upon interactions between a pioneer transcription factor and core histones. Nat Genet 2020;52:418-27.

10. Bassel-Duby R, Hernandez MD, Yang Q, et al. Myocyte nuclear factor, a novel winged-helix transcription factor under both developmental and neural regulation in striated myocytes. Mol Cell Biol 1994;14:4596-605.

11. Garry DJ, Yang Q, Bassel-Duby R, et al. Persistent expression of MNF identifies myogenic stem cells in postnatal muscles. Dev Biol 1997;188:280-94.

12. Shi X, Bowlin KM, Garry DJ. Fhl2 interacts with Foxk1 and corepresses Foxo4 activity in myogenic progenitors. Stem Cells 2010;28:462-9.

13. Shi X, Garry DJ. Sin 3 interacts with Foxk1 and regulates myogenic progenitors. Mol Cell Biochem 2012;366:251-8.

14. Shi X, Seldin DC, Garry DJ. Foxk1 recruits the Sds3 complex and represses gene expression in myogenic progenitors. Biochem J 2012;446:349-57.

15. Meeson AP, Shi X, Alexander MS, et al. Sox15 and Fhl3 transcriptionally coactivate Foxk1 and regulate myogenic progenitor cells. Embo J 2007;26:1902-12.

16. Hawke TJ, Jiang N, Garry DJ. Absence of p21CIP rescues myogenic progenitor cell proliferative and regenerative capacity in Foxk1 null mice. J Biol Chem 2003;278:4015-20.

17. Shi $\mathrm{X}$, Wallis AM, Gerard RD, et al. Foxk1 promotes cell proliferation and represses myogenic differentiation by regulating Foxo4 and Mef2. J Cell Sci 2012;125:5329-37.

18. Garry DJ, Meeson A, Elterman J, et al. Myogenic stem cell function is impaired in mice lacking the forkhead/ winged helix protein MNF. Proc Natl Acad Sci U S A 2000;97:5416-21.

19. Yang X, Hu F, Liu JA, et al. Nuclear DLC1 exerts oncogenic function through association with FOXK1 for cooperative activation of MMP9 expression in melanoma. Oncogene 2020;39:4061-76.

20. Gao F, Tian J. FOXK1, Regulated by miR-365-3p, Promotes Cell Growth and EMT Indicates Unfavorable 
Prognosis in Breast Cancer. Onco Targets Ther 2020;13:623-34.

21. Chen Y, Wu J, Liang G, et al. CHK2-FOXK axis promotes transcriptional control of autophagy programs. Sci Adv 2020;6:eaax5819.

22. Li ZQ, Qu M, Wan HX, et al. FOXK1 promotes malignant progression of breast cancer by activating $\mathrm{PI} 3 \mathrm{~K} /$ AKT/mTOR signaling pathway. Eur Rev Med Pharmacol Sci 2019;23:9978-87.

23. Zhang H, Wu X, Xiao Y, et al. Coexpression of FOXK1

Cite this article as: Garry DJ, Maeng G, Garry MG. Foxk1 regulates cancer progression. Ann Transl Med 2020;8(17):1041. doi: 10.21037/atm-2020-94 and vimentin promotes EMT, migration, and invasion in gastric cancer cells. J Mol Med (Berl) 2019;97:163-76.

24. Xu H, Huang S, Zhu X, et al. FOXK1 promotes glioblastoma proliferation and metastasis through activation of Snail transcription. Exp Ther Med 2018;15:3108-16.

25. Wang Y, Qiu W, Liu N, et al. Forkhead box K1 regulates the malignant behavior of gastric cancer by inhibiting autophagy. Ann Transl Med 2020;8:107. 